\title{
BIBLIOGRAPHY OF W. W. MORGAN
}

1927 (with O. Struve)

(Abstract)

1928 (Abstract)

1930

1931

1932 (with O. Struve)

(with G. Farnsworth)
Orbit of the Spectroscopic Binary 950 Leonis, Astrophys. J. 66, 135.

Spectroscopic and Photometric Observations of CP Cygni, Pop. Astron. 35, 490.

Observations of the Short-Period Variable AK Herculis, Pop. Astron. 35, 547.

Two Recent Solar Eruptions, Pop. Astron. 36, 604.

Leonids Observed at the Yerkes Observatory, Pop. Astron. 38, 624.

Light-Curve and Orbit of the Eclipsing Binary ZZ Aurigae, Pop. Astron. 38, 466.

Studies in Peculiar Stellar Spectra. I. The Manganese Lines in $\alpha$ Andromedae, Astrophys. J. 73, 104.

Studies in Peculiar Stellar Spectra. II. The Spectrum of B. D. $-18^{\circ} 3789$, Astrophys. $J$. 74, 24.

On the Intensities of Stellar Absorption Lines, Proc. Nat. Acad. Sc. Washington 18, 590.

Studies in Peculiar Stellar Spectra. III. On the Occurrence of Europieum in A-Type Stars, Astrophys. J. 75, 46.

On the Variable Lines of Helium and Magnesium in the Spectrum of $13 \mu$ Sagittarii, Astrophys. J. 75, 407.

Note on the Spectrum of $37 \theta$ Aurigae, Astrophys. J. 75, 423.

A Study of the Composite Spectrum of the A-Type Star 14 Comae, Astrophys. J. 76, 144.

Four Early-Type Stars Having Variable Absorption Lines, Astrophys. J. 76, 275.

The Spectrum of the A2 Dwarf $\epsilon$ Serpentis, Astrophys. J. 76, 299. 
1933 (with O. Struve and C. T. Elvey)

1934

(with B. A. Wooten)

1935

(with O. Struve and C. T. Elvey)
Four A- and F-Type Stars Whose Spectra Contain Variable Absorption Lines, Astrophys. J. 76, 315.

Note on the Occultation of BD $+8^{\circ} 2456$ by Jupiter, J. Brit. Astron. Assoc. 43, 325.

A Study of the Spectrum Variable 73 Draconis, Astrophys. J. 77, 77.

On a Line at $\lambda 4470$ in the Spectrum of 21 Aquilae, Astrophys. J. 77, 226.

Some Effects of Changes in Stellar Temperatures and Absolute Magnitudes, Astrophys. J. 77, 291.

Some Evidence for the Existence of a Peculiar Branch of the Spectral Sequence in the Interval B8-F0, Astrophys. J. 77, 330.

On the Relative Intensities of Certain Lines of $\mathrm{Ti}$ II in the Spectra of Three F-Type Stars, Astrophys. J. 78, 158.

A II in the Spectrum of $v$ Sagittarii, Astrophys. J. 79, 513.

Relative Stellar Energy Distribution in the Infrared, Astrophys. J. 80, 229.

A Descriptive Study of the Spectra of The A-Type Stars, Publ. Yerkes Obs. 7, 133.

A Peculiar Spectroscopic Phenomenon in The Algol System, Astrophys. J. 81, 348.

Variations in the Spectrum of 29 Canis Majoris, Astrophys. J. 82, 95.

A Possible Interpretation of the Absorption Spectra of Nova Herculis, Astrophys. J. 83, 252.

A Useful Fine-Grain Developer for Spectrographic Photography, Astrophys. J. 83, 254. On the Spectral Classification of the Stars of Types A to K, Astrophys. J. 85, 380.

Note on a Possible Effect of the Recent Increase in Brightness of $\gamma$ Cassiopeiae, Astrophys. J. 86, 100.

1938
On the Determination of Color Indices of Stars From a Classification of Their Spectra, Astrophys. J. 87, 460 .

On the Spectral Types and Luminosities of 
(with C. T. Elvey)

1939

(with F. Sherman)

(with F. Sherman)

(with P.C. Keenan)

(with W. J. Luyten and O. Struve)

1940 (with J. Titus)

1941 (with P. C. Keenan)

1943 (with P. C. Keenan and E. Kellman)

(Abstract)

1944 (with W. A. Hiltner)

(Abstract)

(Abstract)

1946 (with W. P. Bidelman) the M. Dwarfs, Astrophys. J. 87, 589.

A New Fourth-Magnitude Eclipsing Binary, Astrophys. J. 88, 110.

Storrs B. Barrett, Pop. Astron. 46, 127.

Molecular Bands as Indicators of Stellar Temperatures and Luminosities, Astrophys. J. 89, 310.

On the Color of P Cygni, Astrophys. J. 89, 509.

The Nebulosity near S Monocerotis, Astrophys. J. 89, 553.

On the Spectral Type of the Sun, Publ. Astron. Soc. Pacific 51, 355.

Note on Interstellar Reddening in the Region of $\gamma$ Cygni, Astrophys. J. 90, 632 .

Re-observation of the Orbits of Ten Spectroscopic Binaries with a Discussion of Apsidal Motions, Publ. Yerkes Obs. 7, IV, 251.

On the Classification of the A Stars. I. The Spectral Types of the Brighter Members of the Hyades Cluster, Astrophys. J. 92, 256. The Classification of the Red Carbon Stars, Astrophys. J. 94, 501.

Yerkes Observatory (1897-1941) and Some Notes on the Astronomical Struves, The $S k y$ 5, No. 8.

Some Uses of Spectral Classification, The Telescope 8, 77 and 92.

An Atlas of Stellar Spectra, With An Outline of Spectral Classification, Univ. of Chicago Press.

On the Parallaxes of Some of the Brighter Helium Stars, Publ. Amer. Astron. Soc. 10, 310.

Complex Lines in the Spectrum of $\theta$ Aurigae, Astrophys. J. 99, 318.

The Helium Stars Having the Most Rapid Axial Rotation, Astron. J. 51, 21.

Note on Interstellar Reddening in the Region of the Orion Nebula, Astron. J. 51, 21. New H $\alpha$ Emission Stars, Astrophys. J. 103, 378. 
(with I. Hansen)

(with S. Sharpless)

(with W. P. Bidelman)

1947 (with G. P. Kuiper)

(with A. J. Deutsch)

1948 (with P. C. Keenan and G. Munch) (Abstract)

(with N. G. Roman and

O. J. Eggen)

1949 (with J. J. Nassau)

(Abstract)

1950 (with N. G. Roman)

(with N. G. Roman)

(with J. J. Nassau)

1951

(with J. J. Nassau)

(with H. L. Johnson)

(with W. S. Fitch)

(with P. C. Keenan)

1952 (with J. J. Nassau)
Be Stars Showing Bright Lines of Fe II Astrophys. J. 103, 379.

An Interesting Emission-Line Star Near the Orion Nebula, Astrophys. J. 103, 249.

On the Interstellar Reddening in the Region of the North Polar Sequence and the Normal Color Indices of A-Type Stars, Astrophys. $J$. 104, 245.

Studies of the Stellar System, Science 106, 201.

The Spectrum of $\mathrm{T}$ Coronae Borealis at its 1946 Outburst, Astrophys. J. 106, 362.

Spectra of High-Velocity Giants, Astron. J. 53, 194.

The Classification of the 'Metallic-Line' Stars, Astrophys. J. 107, 107.

A Survey for Stars of High Luminosity in the Galaxy, Astron. J. 54, 192.

The Moving Cluster in Perseus, Astrophys. $J$. 111, 426.

Revised Standards for Supergiants on the System of the Yerkes Spectral Atlas, Astrophys. J. 112, 362.

A Finding List of $\mathrm{O}$ and $\mathrm{B}$ Stars of High Luminosity, Astrophys. J. 113, 141.

Application of the Principle of Natural Groups to the Classification of Stellar Spectra, Publ. Obs. Univ. Michigan 10, 33.

Distribution of Early Type Stars of High Luminosity Near the Galactic Equator, Publ. Obs. Univ. Michigan 10, 43.

On the Color-Magnitude Diagram of the Pleiades, Astrophys. J. 114, 522.

A Useful Luminosity Discriminant in the Late K and Early M Giants, Astrophys. J. 114, 548.

Classification of Stellar Spectra, Ch. 1 in Astrophysics (ed. by J. A. Hynek), McGrawHill.

A Finding List of F Stars of High Luminosity, Astrophys. J. 115, 475. 
(with S. Sharpless and

D. Osterbrock)

(Abstract)

(with A. D. Code and

A. E. Whitford)

(Abstract)

(with J. J. Nassau and V. Blanco)

(Abstract)

1953 (with A. Blaauw)

(with H. L. Johnson)

(with G. Haro)

(with D. L. Harris and

H. L. Johnson)

(with L. Munch)

(with A. E. Whitford and

A. D. Code)

(with Gu. Gonzalez and

Gr. Gonzalez)

(with Gu. Gonzalez and

Gr. Gonzalez)

(with A. Blaauw)

1954 (with H. L. Johnson and N. G. Roman)

(with B. Stromgren and H. M. Johnson)

(Abstract)

(with H. L. Johnson)

(with D. L. Harris and

N. G. Roman)

(with A. Blaauw)
Some Features of Galactic Structure in the Neighborhood of the Sun, Astron. J. 57, 3.

The Reddest B Stars, Astron. J. 57, 8 .

Surface Distribution of Late M Stars in the Milky Way, Astron. J. 57, 21.

Expanding Motions in the Lacerta Aggregate, Astrophys. J. 117, 256.

Fundamental Stellar Photometry for Standards of Spectral Type on the Revised System of the Yerkes Spectral Atlas, Astrophys. J. 117, 313.

Rapid Variables in the Orion Nebula, Astrophys. J. 118, 16.

Some Characteristics of Color Systems, Astrophys. J. 118, 92.

A Probable Clustering of Blue Giants in Cygnus, Astrophys. J. 118, 161.

Studies in Galactic Structure. I. A Preliminary Determination of the Space Distribution of the Blue Giants, Astrophys. J. 118, 318. Blue Giants in the Neighborhood of NGC 6231, Astrophys. J. 118, 323.

Blue Giants in the Direction of the Galactic Center, Astrophys. J. 118, 345.

Note on the Motion and Possible Origin of the O-Type Star HD $34078=$ AE Aurigae and the Emission Nebula IC 405, Bull. Astron. Inst. Neth. 448, 76.

A Very Red Star of Early Type in Cygnus, Publ. Astron. Soc. Pacific 66, 85.

Some Astrometric Problems of Galactic Structure, Astron. J. 59, 86.

New Features of Some Emission Regions in the Milky Way, Astron. J. 59, 188.

A Heavily Obscured O-Association in Cygnus, Astrophys. J. 119, 344.

Photometric and Spectroscopic Observations of Stars in IC 348, Astrophys. J. 119, 622. The Space Motions of AE Aurigae and $\mu$ 
(with J. J. Nassau and

V. M. Blanco)

(with A. B. Meinel and

H. M. Johnson)

1955

(with A. Blaauw and F. C. Bertiau)

(with B. Stromgren and

H. M. Johnson)

(with A. D. Code and

A. E. Whitford)

(with H. L. Johnson)

(with H. L. Johnson)

1956

(with D. L. Harris)

1957 (with N. L. Gould and

G. H. Herbig)

(with N. U. Mayall)

(with H. A. Abt and B. Stromgren)
Columbae with Respect to the Orion Nebula, Astrophys. J. 119, 625.

Reddened Early M- and S-Type Stars Near the Galactic Equator, Astrophys. J. 120, 478.

Spectral Classification with Exceedingly Low Dispersion, Astrophys. J. 120, 506.

La Classification Spectrale de deux Populations Stellaires, In Principes Fondamentaux de Classification Stellaire, Coll. Int. C.N.R.S. (June-July, 1953), 55, 7.

19 Scorpii, a Luminous A Star in the ScorpioCentaurus Association, Astrophys. J. 121, 557.

A Description of Certain Galactic Nebulosities, Astrophys. J. 121, 611.

Studies in Galactic Structure. II. Luminosity Classification for 1270 Blue Giant Stars, Astrophys. J. Suppl. 2, 41.

Some Evidence for a Regional Variation in the Law of Interstellar Reddening, Astrophys. J. 122, 142.

Photometric and Spectroscopic Observations of the Double Cluster in Perseus, Astrophys. J. 122, 429.

Spiral Structure of the Galaxy, Scientific American 192, 42.

The Integrated Spectral Types of Globular Clusters, Publ. Astron. Soc. Pacific 68, 509. The Galactic Cluster M29 (NGC 6913), Vistas in Astronomy 2, 1124.

$\mathrm{BD}+75^{\circ} 325$ : A Subluminous O-Type Star, Publ. Astron. Soc. Pacific 69, 242.

A Spectral Classification of Galaxies, Publ. Astron. Soc. Pacific 69, 291.

A Description of Certain Galactic Nebulosities. II, Astrophys. J. 126, 322.

A Preliminary Classification of the Forms of Galaxies According to their Stellar Population, Publ. Astron. Soc. Pacific 70, 364.

The Observation of Blue Giant Stars at Great Distances from the Sun, In The Large-Scale 
Structure of the Galactic System, IAU Symp. 5, 57.

Systems of Spectroscopic Luminosity Criteria, Astron. J. 63, 180.

Some Characteristics of the Strong- and Weak-Line Stars, In Stellar Populations, Conference, Pontifical Acad. Sci., Vatican City, May 1958, Specola Vat. Richerce Astron. 5, 323.

Some Features of Stellar Populations as Determined From Integrated Spectra. Ibid., 325.

Some Spectroscopic Phenomena Associated with the Stellar Population of Galaxies. In La Structure de l'Evolution de l'Universe,

(Abstract)

1959

(Book review)

1960

1961

1962 11 th Solvay Physics Conf. Brussels, p. 297. On the Stellar Population of the Nuclear Region of the Galaxy. Read at National Acad. Sciences, Nov., 1958, Science 128, 1147.

The Spectra of Galaxies, Publ. Astron. Soc. Pacific 71, 92.

A Preliminary Classification of the Forms of Galaxies According to Their Stellar Population, Publ. Astron. Soc. Pacific 71, 352.

The Integrated Spectra of Globular Clusters, Astron. J. 64, 432.

F. Zwicky: Morphological Astronomy, Publ. Astron. Soc. Pacific 71, 352.

Some Vistas of Astronomical Discovery, Science 132, 73 (Reprinted in Publ. Astron Soc. Pacific 72, 153).

Some Characteristics of Galaxies Which Bear on Their Use as a Fundamental Astrometric Frame of Reference, Astron. J. 65, 222.

The Classification of Clusters of Galaxies, Proc. National Acad. of Sciences, Washington, 47, 905.

Some Characteristics of Galaxies, (The Henry Norris Russell lecture), Astrophys. $J$. 135,1 .

(with T. A. Mathews)

On the Optical Forms of the Brightest Extra- 
(Abstract)

(with N. U. Mayall)

1963 (with W. A. Hiltner)

(Abstract)

(with J. S. Neff)

(Abstract)

1964 (with T. A. Mathews and

M. Schmidt)

(with J. S. Neff)

(Abstract)

1965 (with W. A. Hiltner)

(with W. A. Hiltner and J. S. Neff)

(with W. A. Hiltner, J. S. Neff, R. F. Garrison and D. E. Osterbrock)

(with J. Lesh)

1966

(with K. Lodén)

1967

1968 (with H. J. Smith and

D. Weedman)

(with C. Jaschek, M. Jaschek and A. Slettebak) galactic Radio Sources, Science 138, 992.

Spectral Types of Galaxies, In 'Symposium on Problems of Extra-Galactic Research', IAU Symp. 15 (Santa Barbara), 22.

Spectroscopic Discriminant for O-Type Star Clusters, Astron. J. 68, 281.

Color Separation of Giant and Dwarf Stars, Astron. J. 68, 288.

A Discussion of Galaxies Identified With Radio Sources, Astrophys. J. 140, 35.

Stellar Population of the Nuclear Region of the Andromeda Nebula, Astron. J. 69, 145. Studies in Spectral Classification. I. The HR Diagram of the Hyades, Astrophys. J. 141, 177.

Studies in Spectral Classification. II. The HR Diagram of NGC 6530, Astrophys. J. 141, 183.

Studies in Spectral Classification. III. The HR Diagram of NGC 2244 and NGC 2264, Astrophys. J. 142, 974.

The Supergiant Galaxies, Astrophys. J. 142, 1364.

Introductory Paper. In 'Spectral Classification and Multicolor Photometry', IAU Symp. 24, Saltsjöbaden, Aug. 1964, 3.

Some Characteristics of the Orion Association, Vistas in Astronomy 8, 83.

Frank Elmore Ross. Biographical Memoirs Natl. Acad. Sci., Washington, 39.

A Note on the MK Classification System, In Modern Astrophysics. A memorial to Otto Struve (ed. by M. Hack), Paris, GauthierVillars, p. 83.

Spectral Variations in the Galaxy NGC 4151, Astrophys. J. Letters 152, L113.

A Comparison of the Optical Forms of Certain Seyfert Galaxies with the N-Type Radio Galaxies, Astrophys. J. 153, 27.

On the Spectrum of Bidelman's Helium Variable Star HD 125823, Astrophys. J. Letters 153, L87. 
(with H. A. Abt, A. B. Meinel and J.W. Tapscott)

1969 (with D. E. Osterbrock)

(with H. A. Abt)

(with W. A. Hiltner)

1970

(with L. P. Bautz)

1971 (with D. S. Heeschen and N. R. Walborn)

(with W. A. Hiltner and

R. F. Garrison)

(with N. R. Walborn and

J. W. Tapscott)

1972
An Atlas of Low-Dispersion Grating Stellar Spectra, Kitt Peak Nat. Obs., Tucson.

Large Scale Distribution of H II Regions in Galaxies, In Interstellar Ionized Hydrogen (ed. by Y. Terzian), W. A. Benjamin, N. Y., 565 .

On the Classification of the Forms and the Stellar Content of Galaxies, Astron. J. 74, 515.

The HR Diagram of NGC 2516. Astron. J. 74, 813 .

UBV Photometry and Spectral Types in NGC 6611, Astron. J. 74, 1152.

Struve's Approach to Spectral Classification, In Spectroscopic Astrophysics (ed. by G. H. Herbig), Univ. of California Press, Berkeley, p.25.

Spiral Structure in External Galaxies, In 'The Spiral Structure of Our Galaxy', IAU Symp. 38 (Basel), 9.

On the Classification of the Forms of Clusters of Galaxies, Astrophys. J. 162, L149.

The Distribution of Light in the Central Regions of Some Giant Elliptical Galaxies, Astrophys. J. 165, L65.

The HR Diagram of the Alpha Persei Cluster, Astron. J. 76, 242.

An Optical Form Morphology of Seyfert Galaxies, In Study Week on Nuclei of Galaxies (April 13-18, 1970). (ed. by D. J. O'Connell), Pontif. Acad. Sci. Scripta Varia 35, 27.

A Unitary Classification for $\mathrm{N}$ Galaxies, Astron. J. 76, 1000.

Classification of Compact Objects: QSS, QSO's, N-Type and Compact Galaxies, Seyfert and Galactic Nuclei, In External Galaxies and Quasi-Stellar Objects'. (ed. by D. S. Evans). Uppsala, Aug. 1970, IAU Symp. 44, 97.

The Spectral Classification of the F Stars of

(with H. A. Abt) 
(with H. A. Abt)

1973 (with R. A. White and

J. W. Tapscott)

(with H. A. Abt)

(with P. C. Keenan)

1975 (with S. Kayser and R. A. White) (with H. A. Abt)
Intermediate Luminosity, Astron. J. 77, 35. The HR Diagram of the Open Cluster IC 2602, Astrophys. J. 174, L131.

A New Shell Phase in Pleione, Astron. J. 78, 302.

On the Metallicity of the Main-Sequence Stars in M67, Astron. J. 78, 386.

Spectral Classification, Ann. Rev. Astron. Astrophys. 11, 29.

cD Galaxies in Poor Clusters. (in press).

MK Morphology of a Group of Am Stars, Astrophys. J. (in press).

Remarks on Some Aspects of Spectral Classification, IAU Symp. 72. 